Journal of the Operations Research

Society of Japan

Vol. 42, No. 4, December 1999

\title{
OPTIMAL SEQUENTIAL BACKUP STRATEGY UNDER CONSTRAINED RESOURCES
}

\author{
Toshio Hamada \\ Kobe Unversity of Commerce
}

(Received October 9, 1998; Revised March 17, 1999)

\begin{abstract}
When we construct a program by using a computer, we sometimes interrupt to work and store the program constructed up to this point on a disk or another medium. At that time, we sometimes make a backup copy of the program on another disk. Whether we make a backup copy or not seems to depend on the probability that the program on a disk will be broken by an accident during the work. This kind of problem is formulated by dynamic programming and the optimal strategy is derived with the critical values whose explicit form is analytically derived.
\end{abstract}

\section{Introduction}

We consider a disk or another medium on which we can not overwrite on it once we store programs or data on it. This sometimes occurs because of the physical reason or because of the reason we keep the histrical files without being overwritten. As the matter of convenience, we call this kind of medium as a disk in this paper. Furthermore, we assume that the recovery of the current program or data is very expensive.

Consider the model as follows: Suppose that there are $n$ new disks and an additional disk called $D_{0}$ on which there is a master file. After we revise the master file, we always store the revised master file on one of new disks called $D_{1}$ as a version 1 . At this point of time, we have to decide whether to make an additional copy (backup copy) of disk or not. We call the decision to make a backup copy as action $a_{2}$ and the decision not to make it as action $a_{1}$. If $a_{1}$ is selected, the number of the remaining new disks becomes $n-1$. If $a_{2}$ is selected, we store the revised master file on an additional new disk as a backup and the number of the remaining new disks becomes $n-2$. Once a new disk is used to store a master file, we assume that it will not be used again in the future in order to store another new version of the master file.

Assume that $a_{1}$ is made. Then, we begin to revise the master file of version 1 on $D_{1}$ and after the work of revision we store it on a new disk called $D_{2}$ as version 2 . If an accident occurs before saving it on $D_{2}$ and the file of revised master file on $D_{1}$ is broken, we have to go back to the starting point. In this case, we have to stop the work because of the assumption that the recovery cost is very expensive. Let $p$ be the probability that the floppy disk (or the master file on the floppy disk) will be broken during the revising work. Then, the probability that no accident occurs before saving the revised master file is $1-p$ and in this case of no accidents we continue to work and have to select one of two actions, $a_{1}$ or $a_{2}$, as a decision.

Assume that we select $a_{2}$ at this point of time. Then, the revised master file is saved on two new disks called $D_{3}$ and $D_{4}$. At the next stage, we begin to revise the master file on $D_{3}$ and after some work of revision, we save it on a new disk $D_{5}$. But if an accident occurs in 
this point of time, we can continue to repeat the revising work by using $D_{4}$. In this case, if the accident occurs once more during the work of using $D_{4}$, we will not be able to continue to revise the current master file and therefore we will give up to continue the work. As long as the current master file is available, we can continue to revise it to make a new version of the disk. The objective is to maximize the expected value of the number of the last version of the master file which we can attain by starting with $n$ new floppy disks and the master file of version 0 .

Checkpointing is also an important strategy to make the recovery work and have been studied in many papers, for example, Chandy and Ramamoorthy [2], Young [14], Gelenbe [6], Toueg and Babaog̃lu [13], Kaio and Osaki [8], Dohi, Aoki, Kaio and Osaki [3], and so on. Whether to make a backup copy of the hard computer disks or not is also the important problem in the data processing and some backup policies for that problem have been discussed in Sandoh and Kawaii [11], Sandoh, Kaio and Kawaii [10], and Sandoh, Kawaii and Ibaraki [12]. Fukumoto, Yasui and Nakagawa [5] discuss the Markov chain model to evaluate two kinds of backup strategies for the second storage. Hamada [7] has considered that the decision point that there are $n$ files to be made in the future, that the first one of them has just been made under the condition that there are $k$ files whose backup copies do not exist, and that there remains a sufficiently large memory space. Both the cost of making a backup copy and that of losing the file whose backup copy does not exist are also considered with the reward evaluating the safety of a file which is obtained by making its backup copy. This kind of problem is considered as the sequential decision problem with a finite horizon and discussed an optimal backup strategy by using stochastic dynamic programming (See, for example, Ross [9]).

In this paper, we derive the optimal sequential backup strategy under a constraint of finite number of resources. The model considered in this paper is useful not only in the backup of files but also in the several areas where it is important to keep the lifetime of a system as long as possible by replacing a part under the condition that the part will be broken with a positive probability $p$ and the backup policy is useful. In Section 2 , the problem is formulated by dynamic programming and the optimal strategy is derived in Section 3. The model is extended to the case that the value of the parameter is unknown and some properties of the optimal strategy are derived.

\section{Formulation by Dynamic Programming}

Let $n$ be the number of the remaining new disks and $p$ be the probability that the accident occurs during the revising work. Then, the state is denoted by $n$ if $p$ is known. Let $a_{1}$ be the action not to make a backup file of the current disk and $a_{2}$ the action to make a backup copy. At the state $n$ with $n \geq 2$, one of two actions $a_{1}$ and $a_{2}$ is selected. If $a_{1}$ is selected at state $n$, the transition probability from the state $n$ to the state $n-1$ is $1-p$, that from the state $n$ to the state 0 (the absorbing state) is $p$, and that from the state $n$ to all the other states is 0 . In this case, the reward 1 is obtained only when the state changes from $n$ to $n-1$. If $a_{2}$ is selected at state $n$, the transition probability from the state $n$ to the state $n-2$ is $1-p^{2}$, that from the state $n$ to the state 0 is $p^{2}$, and that from the state $n$ to all the other state is 0 . In this case, the reward 1 is obtained only when the state changes from $n$ to $n-2$. The objective is to maximize the total expected reward obtained up to reach the state 0 , which is also equal to the total expected number of revisions up to reach the state 0 .

Let $f_{n}(p)$ be the maximum expected reward when there are $n$ new disks available and the current version is 0 . Also, let $f_{n}^{i}(p)(i=1,2)$ be the maximum expected reward when 
there are $n$ unused disks available and the current version is 0 if $a_{i}$ is selected at the first stage and the optimal strategy is followed thereafter. Then,

$$
\begin{gathered}
f_{0}(p)=0 \\
f_{1}(p)=1-p
\end{gathered}
$$

and

for $n=2,3,4, \cdots$, where

$$
f_{n}(p)=\max \left\{f_{n}^{1}(p), f_{n}^{2}(p)\right\}
$$

$$
\begin{aligned}
f_{n}^{1}(p) & =(1-p)\left(1+f_{n-1}(p)\right)+p \times 0 \\
& =(1-p)\left(1+f_{n-1}(p)\right)
\end{aligned}
$$

and

$$
\begin{aligned}
f_{n}^{2}(p) & =\left(1-p^{2}\right)\left(1+f_{n-2}(p)\right)+p^{2} \times 0 \\
& =\left(1-p^{2}\right)\left(1+f_{n-2}(p)\right)
\end{aligned}
$$

for $n \geq 2$. Then, in state $n, a_{1}$ is optimal if and only if $f_{n}^{1}(p) \geq f_{n}^{2}(p)$. For $n=2$,

$$
f_{2}^{1}(p)=(1-p)(2-p)
$$

and

$$
f_{2}^{2}(p)=1-p^{2}
$$

and therefore

$$
f_{2}(p)= \begin{cases}(1-p)(2-p), & \text { if } 0<p \leq p_{2} \\ 1-p^{2}, & \text { if } p_{2}<p<1\end{cases}
$$

where

$$
p_{2}=\frac{1}{2}
$$

For $n=3$,

$$
f_{3}^{1}(p)= \begin{cases}(1-p)\{1+(1-p)(2-p)\}, & \text { if } 0<p \leq p_{2} \\ (1-p)\left(2-p^{2}\right), & \text { if } p_{2}<p<1\end{cases}
$$

and

$$
f_{3}^{2}(p)=(1-p)(1+p)(2-p)
$$

and therefore

$$
f_{3}(p)= \begin{cases}(1-p)\{1+(1-p)(2-p)\}, & \text { if } 0<p \leq p_{3} \\ (1-p)(1+p)(2-p), & \text { if } p_{3}<p<1\end{cases}
$$

where

$$
p_{3}=\frac{2-\sqrt{2}}{2}
$$

Now, let

$$
d_{n}(p)=f_{n}^{1}(p)-f_{n}^{2}(p)
$$

for $n \geq 2$. Then, the following lemma is derived. 
Lemma 1 For $0<p<1$,

$$
\begin{gathered}
d_{2}(p)=(1-p)(1-2 p) \\
d_{3}(p)= \begin{cases}(1-p)\left(1-4 p+2 p^{2}\right), & \text { if } 0<p \leq p_{2} \\
-p(1-p), & \text { if } p_{2}<p<1\end{cases}
\end{gathered}
$$

and

$$
d_{n}(p)=(1-p)\left[-p+\max \left\{d_{n-1}(p), 0\right\}+(1+p) \min \left\{d_{n-2}(p), 0\right\}\right]
$$

for $n \geq 4$.

Proof. (2.10) is derived from (2.3) and (2.4) and $d_{2}(p) \geq 0$ if and only if $0<p \leq 1 / 2$, that is, $f_{2}^{1}(p) \geq f_{2}^{2}(p)$ if and only if $0<p \leq 1 / 2$. For $n=3,(2.11)$ is derived from (2.6) and (2.7). For $n \geq 4$, from (2.1), (2.2) and (2.9),

$$
d_{n}(p)=(1-p)\left(1+f_{n-1}(p)\right)-\left(1-p^{2}\right)\left(1+f_{n-2}(p)\right),
$$

where

$$
\begin{aligned}
f_{n-1}(p) & =\max \left\{f_{n-1}^{1}(p), f_{n-1}^{2}(p)\right\} \\
& =f_{n-1}^{2}(p)+\max \left\{f_{n-1}^{1}(p)-f_{n-1}^{2}(p), 0\right\} \\
& =\left(1-p^{2}\right)\left\{1+f_{n-3}(p)\right\}+\max \left\{d_{n-1}(p), 0\right\}
\end{aligned}
$$

and

$$
\begin{aligned}
f_{n-2}(p) & =\max \left\{f_{n-2}^{1}(p), f_{n-2}^{2}(p)\right\} \\
& =f_{n-2}^{1}(p)+\max \left\{0,-\left(f_{n-2}^{1}(p)-f_{n-2}^{2}(p)\right)\right\} \\
& =(1-p)\left\{1+f_{n-3}(p)\right\}-\min \left\{d_{n-2}(p), 0\right\} .
\end{aligned}
$$

Therefore

$$
\begin{aligned}
d_{n}(p)= & (1-p)\left[1+\left(1-p^{2}\right)\left\{1+f_{n-3}(p)\right\}+\max \left\{d_{n-1}(p), 0\right\}\right] \\
& -\left(1-p^{2}\right)\left[1+(1-p)\left\{1+f_{n-3}(p)\right\}-\min \left\{d_{n-2}(p), 0\right\}\right] \\
= & (1-p)\left[-p+\max \left\{d_{n-1}(p), 0\right\}+(1+p) \min \left\{d_{n-2}(p), 0\right\}\right]
\end{aligned}
$$

This completes the proof. $\square$

Lemma 2 For $n \geq 1$,

$$
\lim _{p \rightarrow 0+} f_{n}(p)=n
$$

and

$$
\lim _{p \rightarrow 1-} f_{n}(p)=0
$$

and for $n \geq 2$,

$$
\lim _{p \rightarrow 0+} d_{n}(p)=1 .
$$

Proof. As $f_{1}(p)=1-p$, both (2.13) and (2.14) hold for $n=1$. For $n=2$, both (2.13) and (2.14) are derived from (2.5). From (2.10),

$$
\begin{aligned}
\lim _{p \rightarrow 0+} d_{2}(p) & =\lim _{p \rightarrow 0+}(1-p)(1-2 p) \\
& =1
\end{aligned}
$$


and therefore (2.15) holds for $n=2$. Suppose that (2.13), (2.14) and (2.15) hold for $n=k \geq 2$. Since

$$
\begin{gathered}
f_{k+1}^{1}(p)=(1-p)\left(1+f_{k}(p)\right) \\
f_{k+1}^{2}(p)=\left(1-p^{2}\right)\left(1+f_{k-1}(p)\right)
\end{gathered}
$$

and

$$
d_{k+1}(p)=f_{k+1}^{1}(p)-f_{k+1}^{2}(p)
$$

(2.13), (2.14) and (2.15) hold for $n=k+1$. This completes the proof. $\square$

\section{Optimal Strategy}

Now, let

$$
p_{1}=1
$$

and

$$
p_{n}=1-\left(\frac{1}{2}\right)^{\frac{1}{n-1}}
$$

for $n \geq 2$. Then,

$$
\left(\frac{1}{2}\right)^{\frac{1}{n-1}}<\left(\frac{1}{2}\right)^{\frac{1}{n}}
$$

and therefore the monotonicity property that $p_{n}>p_{n+1}$ for $n \geq 1$ is easily derived.

Theorem 1 (i) For $0<p \leq p_{n-1}$,

$$
f_{n}^{1}(p)=\frac{1-p}{p}\left\{1-(1-p)^{n}\right\}
$$

and

$$
f_{n}^{2}(p)=\frac{1-p^{2}}{p}\left\{1-(1-p)^{n-1}\right\}
$$

(ii) for $0<p \leq p_{n-1}$,

$$
d_{n}(p)=2(1-p)\left\{(1-p)^{n-1}-\frac{1}{2}\right\}
$$

(iii) $d_{n}(p) \geq 0$ if and only if $0<p \leq p_{n}$,

(iv) $f_{n}(p)=f_{n}^{1}(p)$ if $0<p \leq p_{n}$.

Proof. (2.3), (2.4) and (2.10) are rewritten respectively as follows:

$$
\begin{aligned}
& f_{2}^{1}(p)=\frac{1-p}{p}\left\{1-(1-p)^{2}\right\} \\
& f_{2}^{2}(p)=\frac{1-p^{2}}{p}\{1-(1-p)\}
\end{aligned}
$$

and

$$
d_{2}(p)=2(1-p)\left\{(1-p)-\frac{1}{2}\right\} \text {. }
$$

Since these equations hold for $0<p \leq p_{1}$, both (i) and (ii) hold for $n=2$. Also, for $n=2$, (iii) is derived from (2.10) and also (iv) is the immediate consequence of (iii). For $n=3$, it is derived from (2.6) and (2.7) that

$$
f_{3}^{1}(p)=\frac{1-p}{p}\left\{1-(1-p)^{3}\right\}
$$


and

$$
f_{3}^{2}(p)=\frac{1-p^{2}}{p}\left\{1-(1-p)^{2}\right\}
$$

for $0<p \leq p_{2}$, and it is derived from (2.11) that

$$
d_{3}(p)= \begin{cases}2(1-p)\left\{(1-p)^{2}-\frac{1}{2}\right\}, & \text { if } 0<p \leq p_{2} \\ -p(1-p), & \text { if } \quad p_{2}<p<1\end{cases}
$$

from which $d_{3}(p) \geq 0$ if and only if $0<p \leq p_{3}$ and therefore $f_{3}(p)=f_{3}^{1}(p)$ if $0<p \leq p_{3}$, that is, (iii) and (iv) hold for $n=3$. For $k \geq 3$, suppose that (i), (ii), (iii) and (iv) hold for $n \leq k$. Then

$$
f_{k}(p)=f_{k}^{1}(p)
$$

for $0<p \leq p_{k}$ and

$$
f_{k-1}(p)=f_{k-1}^{1}(p)
$$

for $0<p \leq p_{k-1}$. From inductive hypothesis and $p_{k}<p_{k-1}$,

$$
\begin{aligned}
f_{k+1}^{1}(p) & =(1-p)\left(1+f_{k}^{1}(p)\right) \\
& =(1-p)\left[1+\frac{1-p}{p}\left\{1-(1-p)^{k}\right\}\right] \\
& =\frac{1-p}{p}\left\{1-(1-p)^{k+1}\right\}
\end{aligned}
$$

and

$$
\begin{aligned}
f_{k+1}^{2}(p) & =\left(1-p^{2}\right)\left(1+f_{k-1}^{1}(p)\right) \\
& =\frac{1-p^{2}}{p}\left\{1-(1-p)^{k}\right\}
\end{aligned}
$$

for $0<p \leq p_{k}$, from which

$$
\begin{aligned}
d_{k+1}(p) & =f_{k+1}^{1}(p)-f_{k+1}^{2}(p) \\
& =2(1-p)\left\{(1-p)^{k}-\frac{1}{2}\right\}
\end{aligned}
$$

for $0<p \leq p_{k}$. Therefiore, (i) and (ii) hold for $n=k+1$. (3.1) means that $d_{k+1}(p) \geq 0$ if $0<p \leq p_{k+1}$. Since $d_{k}(p) \geq 0$ if and only if $0<p \leq p_{k}$ and $d_{k-1}(p)<0$ if and only if $p_{k-1}<p<1$,

$$
d_{k+1}(p)=\left\{\begin{array}{lll}
2(1-p)\left\{(1-p)^{k}-\frac{1}{2}\right\}, & \text { if } 0<p \leq p_{k} \\
-p(1-p), & \text { if } p_{k}<p \leq p_{k-1} \\
(1-p)\left\{-p+(1+p) d_{k-1}(p)\right\}, & \text { if } p_{k-1}<p<1
\end{array}\right.
$$

is derived from $(2.12),(3.1)$ and the inequality $p_{k}<p_{k-1}$. Therefore, $d_{k+1}(p) \geq 0$ if and only if $0<p \leq p_{k+1}$, which means that $f_{k+1}(p)=f_{k+1}^{1}(p)$ for $0<p \leq p_{k+1}$. This completes the proof. $\square$

This theorem gives the optimal strategy:

Optimal strategy: For state $n, a_{2}$ is optimal if and only if $p>p_{n}$.

The values of $p_{n}$ for $n=1,2, \cdots, 10$ are given in Table 1 . 
Table 1: Values of $p_{n}$ for $n=1,2, \cdots, 10$

\begin{tabular}{ccccccccccc}
\hline$n$ & 1 & 2 & 3 & 4 & 5 & 6 & 7 & 8 & 9 & 10 \\
\hline$p_{n}$ & 1.0000 & 0.5000 & 0.2929 & 0.2063 & 0.1591 & 0.1294 & 0.1091 & 0.0943 & 0.0830 & 0.0741 \\
\hline
\end{tabular}

\section{Case That $p$ Is Unknown}

Suppose that the values of $p$ is unknown and there is the prior information that $p$ has the beta density function $g(p \mid s, t)$ as the prior information, that is,

$$
g(p \mid s, t)= \begin{cases}\frac{\Gamma(s+t)}{\Gamma(s) \Gamma(t)} p^{s-1}(1-p)^{t-1}, & \text { for } 0<p<1 \\ 0, & \text { otherwise. }\end{cases}
$$

Since beta distribution is the conjugate prior distribution for the unknown parameter of Bernoulli distribution, the posterior distribution after obtaining an observation is also beta distribution (See, for example, DeGroot [4]). The following equations hold for $s>0$ and $t>0$ :

$$
\int_{0}^{1}(1-p) g(p \mid s, t) d p=\frac{t}{s+t}
$$

and

$$
\int_{0}^{1} p(1-p) g(p \mid s, t) d p=\frac{s t}{(s+t)(s+t+1)} .
$$

In this case, the state is specified by $(n ; s, t)$, where $(s, t)$ is the parameters of the current prior distribution and $n$ is the number of the remaining new disks available. In state $(n ; s, t)$, if an acident occurs, the next state is $(n-1 ; s+1, t)$ and if no accident occurs, the state is $(n-1 ; s, t+1)$.

Now, let $F_{n}(s, t)$ be the maximum expected number of the revised version of the program when there are $n$ new disks available and the current information about $p$ is $(s, t)$. Also, let $F_{n}^{i}(s, t)(i=1,2)$ be the maximum expected number of the revised version of the program when there are $n$ new disks available, the current information about $p$ is $(s, t), a_{i}$ is selected at the first stage, and the optimal strategy is followed thereafter. Then,

$$
\begin{gathered}
F_{0}(s, t)=0, \\
F_{1}(s, t)=\frac{t}{s+t}
\end{gathered}
$$

and

$$
F_{n}(s, t)=\max \left\{F_{n}^{1}(s, t), F_{n}^{2}(s, t)\right\},
$$

for $s>0, t>0$ and $n=2,3, \cdots$, where

$$
\begin{aligned}
F_{n}^{1}(s, t) & =\int_{0}^{1}\left\{(1-p)\left(1+F_{n-1}(s, t+1)\right)+p \times 0\right\} g(p \mid s, t) d p \\
& =\frac{t}{s+t}\left(1+F_{n-1}(s, t+1)\right)
\end{aligned}
$$

and

$$
F_{n}^{2}(s, t)=\int_{0}^{1}\left\{(1-p)\left(1+F_{n-2}(s, t+1)\right)\right.
$$




$$
\begin{aligned}
& \left.+p(1-p)\left(1+F_{n-2}(s+1, t+1)\right)+p^{2} \times 0\right\} g(p \mid s, t) d p \\
= & \frac{t}{s+t}\left(1+F_{n-2}(s, t+1)\right)+\frac{t s}{(s+t)(s+t+1)}\left(1+F_{n-2}(s+1, t+1)\right) .
\end{aligned}
$$

$a_{1}$ is optimal if $F_{n}^{1}(s, t) \geq F_{n}^{2}(s, t)$ and $a_{2}$ is optimal if $F_{n}^{1}(s, t) \leq F_{n}^{2}(s, t)$. Both $a_{1}$ and $a_{2}$ are optimal if $F_{n}^{1}(s, t)=F_{n}^{2}(s, t)$. As the matter of convenience, $a_{1}$ is said to be optimal if and only if $F_{n}^{1}(s, t) \geq F_{n}^{2}(s, t)$.

For $n=2$,

$$
\begin{aligned}
F_{2}^{1}(s, t) & =\frac{t}{s+t}+\frac{t(t+1)}{(s+t)(s+t+1)} \\
& =\sum_{k=1}^{2} \prod_{j=1}^{k} \frac{t+j-1}{s+t+j-1}
\end{aligned}
$$

and

$$
\begin{aligned}
F_{2}^{2}(s, t) & =\frac{t}{s+t}+\frac{t s}{(s+t)(s+t+1)} \\
& =\sum_{k=1}^{1} \prod_{j=1}^{k} \frac{t+j-1}{s+t+j-1}\left(1+\frac{s}{s+t+k}\right)
\end{aligned}
$$

that is,

$$
F_{2}(s, t)= \begin{cases}\sum_{k=1}^{2} \prod_{j=1}^{k} \frac{t+j-1}{s+t+j-1}, & \text { if } 0<s \leq r_{2}(t) \\ \sum_{k=1}^{1} \prod_{j=1}^{k} \frac{t+j-1}{s+t+j-1}\left(1+\frac{s}{s+t+k}\right), & \text { if } r_{2}(t)<s\end{cases}
$$

where

$$
r_{2}(t)=t+1 \text {. }
$$

For $n=3$, since

$$
F_{3}^{1}(s, t)=\frac{t}{s+t}\left(1+F_{2}(s, t+1)\right)
$$

and

$$
F_{2}(s, t+1)= \begin{cases}\sum_{k=2}^{3} \prod_{j=2}^{k} \frac{t+j-1}{s+t+j-1}, & \text { if } 0<s \leq r_{2}(t+1), \\ \sum_{k=2}^{2} \prod_{j=2}^{k} \frac{t+j-1}{s+t+j-1}\left(1+\frac{s}{s+t+k}\right), & \text { if } r_{2}(t+1)<s\end{cases}
$$

$F_{3}^{1}(s, t)$ is rewritten as follows:

$$
F_{3}^{1}(s, t)= \begin{cases}\sum_{k=1}^{3} \prod_{j=1}^{k} \frac{t+j-1}{s+t+j-1}, & \text { if } 0<s \leq r_{2}(t+1), \\ \sum_{k=1}^{2} \prod_{j=1}^{k} \frac{t+j-1}{s+t+j-1}\left(1+\frac{s}{s+t+k}\right)-\frac{t s}{(s+t)(s+t+1)}, & \text { if } r_{2}(t+1)<s .\end{cases}
$$


Also, since

$$
F_{3}^{2}(s, t)=\frac{t}{s+t}\left(1+F_{1}(s, t+1)\right)+\frac{t s}{(s+t)(s+t+1)}\left(1+F_{1}(s+1, t+1)\right)
$$

with

$$
F_{1}(s, t+1)=\frac{t+1}{s+t+1}
$$

and

$$
F_{1}(s+1, t+1)=\frac{t+1}{s+t+2}
$$

$F_{3}^{2}(s, t)$ is rewritten as follows:

$$
F_{3}^{2}(s, t)=\sum_{k=1}^{2} \prod_{j=1}^{k} \frac{t+j-1}{s+t+j-1}\left(1+\frac{s}{s+t+k}\right) .
$$

From (4.3) and (4.4),

$$
F_{3}^{1}(s, t)-F_{3}^{2}(s, t)= \begin{cases}\frac{t}{s+t}\left(2 \prod_{j=1}^{2} \frac{t+j}{s+t+j}-1\right), & \text { if } 0<s \leq r_{2}(t+1), \\ -\frac{t s}{(s+t)(s+t+1)}, & \text { if } r_{2}(t+1)<s\end{cases}
$$

$F_{3}^{1}(s, t)-F_{3}^{2}(s, t)<0$ for $r_{2}(t+1)<s, \lim _{s \rightarrow+0}\left(F_{3}^{1}(s, t)-F_{3}^{2}(s, t)\right)=1$, and $2 \prod_{j=1}^{2} \frac{t+j}{s+t+j}-1$ is strictly decreasing in $s$. Hence, the following equation of $s$ has a unique root $r_{3}(t)$ in the interval $\left(0, r_{2}(t+1)\right)$ :

$$
2 \prod_{j=1}^{2} \frac{t+j}{s+t+j}-1=0 .
$$

Since

$$
2 \prod_{j=1}^{2} \frac{t+j}{t+t+j}-1=\frac{1-t}{2 t+1}<0
$$

if $t>1, r_{3}(t)<t$. Also, $F_{3}(s, t)$ is rewritten as follows:

$$
F_{3}(s, t)= \begin{cases}\sum_{k=1}^{3} \prod_{j=1}^{k} \frac{t+j-1}{s+t+j-1}, & \text { if } 0<s \leq r_{3}(t), \\ \sum_{k=1}^{2} \prod_{j=1}^{k} \frac{t+j-1}{s+t+j-1}\left(1+\frac{s}{s+t+k}\right), & \text { if } r_{3}(t)<s\end{cases}
$$

For $n=4$, since

$$
F_{4}^{1}(s, t)=\frac{t}{s+t}\left(1+F_{3}(s, t+1)\right)
$$

and

$$
F_{3}(s, t+1)= \begin{cases}\sum_{k=2}^{4} \prod_{j=2}^{k} \frac{t+j-1}{s+t+j-1}, & \text { if } 0<s \leq r_{3}(t+1), \\ \sum_{k=2}^{3} \prod_{j=2}^{k} \frac{t+j-1}{s+t+j-1}\left(1+\frac{s}{s+t+k}\right), & \text { if } r_{3}(t+1)<s\end{cases}
$$


$F_{4}^{1}(s, t)$ is derived as follows:

$$
F_{4}^{1}(s, t)= \begin{cases}\sum_{k=1}^{4} \prod_{j=1}^{k} \frac{t+j-1}{s+t+j-1}, & \text { if } 0<s \leq r_{3}(t+1), \\ \sum_{k=1}^{3} \prod_{j=1}^{k} \frac{t+j-1}{s+t+j-1}\left(1+\frac{s}{s+t+k}\right)-\frac{t s}{(s+t)(s+t+1)}, & \text { if } r_{3}(t+1)<s .\end{cases}
$$

Also, since

$$
F_{4}^{2}(s, t)=\frac{t}{s+t}\left(1+F_{2}(s, t+1)\right)+\frac{t s}{(s+t)(s+t+1)}\left(1+F_{2}(s+1, t+1)\right)
$$

with

$$
F_{2}(s, t+1)= \begin{cases}\sum_{k=2}^{3} \prod_{j=2}^{k} \frac{t+j-1}{s+t+j-1}, & \text { if } 0<s \leq r_{2}(t+1), \\ \sum_{k=2}^{2} \prod_{j=2}^{k} \frac{t+j-1}{s+t+j-1}\left(1+\frac{s}{s+t+k}\right), & \text { if } r_{2}(t+1)<s\end{cases}
$$

and

$$
F_{2}(s+1, t+1)= \begin{cases}\sum_{k=2}^{3} \prod_{j=2}^{k} \frac{t+j-1}{(s+1)+t+j-1}, & \text { if } 0<s \leq r_{2}(t+1)-1, \\ \sum_{k=2}^{2} \prod_{j=2}^{k} \frac{t+j-1}{(s+1)+t+j-1}\left(1+\frac{s+1}{(s+1)+t+k}\right) & \text { if } r_{2}(t+1)-1<s\end{cases}
$$

$F_{4}^{2}(s, t)$ is derived as follows:

$$
F_{4}^{2}(s, t)= \begin{cases}\sum_{k=1}^{3} \prod_{j=1}^{k} \frac{t+j-1}{s+t+j-1}\left(1+\frac{s}{s+t+k}\right), & \text { if } 0<s \leq r_{2}(t+1)-1, \\ \sum_{k=1}^{3} \prod_{j=1}^{k} \frac{t+j-1}{s+t+j-1}\left(1+\frac{s}{s+t+k}\right) & \\ +\frac{t s(t+1)(s-t-1)}{(s+t)(s+t+1)(s+t+2)(s+t+3)}, & \text { if } r_{2}(t+1)-1<s \leq r_{2}(t+1), \\ \sum_{k=1}^{3} \prod_{j=1}^{k} \frac{t+j-1}{s+t+j-1}\left(1+\frac{s}{s+t+k}\right) & \\ +\frac{t s(t+1)(s-t-1)}{(s+t)(s+t+1)(s+t+2)(s+t+3)} & \\ +\frac{t(t+1)(s-t-2)}{(s+t)(s+t+1)(s+t+2)}, & \text { if } r_{2}(t+1)<s .\end{cases}
$$

Since $r_{2}(t+1)-1=t+1$ and $r_{3}(t+1)<t+1$,

$$
r_{3}(t+1)<r_{2}(t+1)-1 \text {. }
$$


For $0<s \leq r_{3}(t+1)$

$$
F_{4}^{1}(s, t)-F_{4}^{2}(s, t)=\frac{t}{s+t}\left(2 \prod_{j=1}^{3} \frac{t+j}{s+t+j}-1\right) .
$$

Also from the definition of $r_{3}(t+1)$,

$$
2 \prod_{j=1}^{2} \frac{(t+1)+j}{r_{3}(t+1)+(t+1)+j}-1=0
$$

or

$$
2 \prod_{j=2}^{3} \frac{t+j}{r_{3}(t+1)+t+j}-1=0
$$

from which

$$
2 \prod_{j=1}^{3} \frac{t+j}{r_{3}(t+1)+t+j}-1<0
$$

Since $2 \prod_{j=1}^{3} \frac{t+j}{s+t+j}-1$ is decreasing in $s$ in the interval $\left(0, r_{3}(t+1)\right], F_{4}^{1}(s, t)-F_{4}^{2}(s, t)$ is continuous in $s$ in the interval $\left(0, r_{3}(t+1)\right), \lim _{s \rightarrow+0}\left(F_{4}^{1}(s, t)-F_{4}^{2}(s, t)\right)=1$ and $F_{4}^{1}\left(r_{3}(t+1), t\right)-F_{4}^{2}\left(r_{3}(t+1), t\right)<0$, the equation $F_{4}^{1}(s, t)-F_{4}^{2}(s, t)=0$ of $s$,

$$
2 \prod_{j=1}^{3} \frac{t+j}{s+t+j}-1=0
$$

has a unique root $r_{4}(t)$ in the interval $\left(0, r_{3}(t+1)\right)$ and

$$
F_{4}(s, t)= \begin{cases}\sum_{k=1}^{4} \prod_{j=1}^{k} \frac{t+j-1}{s+t+j-1}, & \text { if } 0<s \leq r_{4}(t) \\ \sum_{k=1}^{3} \prod_{j=1}^{k} \frac{t+j-1}{s+t+j-1}\left(1+\frac{s}{s+t+k}\right), & \text { if } r_{4}(t)<s \leq r_{2}(t+1)-1 \\ \sum_{k=1}^{3} \prod_{j=1}^{k} \frac{t+j-1}{s+t+j-1}\left(1+\frac{s}{s+t+k}\right) & \\ +\frac{t s(t+1)(s-t-1)}{(s+t)(s+t+1)(s+t+2)(s+t+3)}, & \text { if } r_{2}(t+1)-1<s \leq r_{2}(t+1) \\ \sum_{k=1}^{3} \prod_{j=1}^{k} \frac{t+j-1}{s+t+j-1}\left(1+\frac{s}{s+t+k}\right) & \\ +\frac{t s(t+1)(s-t-1)}{(s+t)(s+t+1)(s+t+2)(s+t+3)} & \\ +\frac{t(t+1)(s-t-2)}{(s+t)(s+t+1)(s+t+2)}, & \text { if } r_{2}(t+1)<s .\end{cases}
$$

Now, let

$$
D_{n}(s, t)=F_{n}^{1}(s, t)-F_{n}^{2}(s, t)
$$

for $n \geq 2$. Then, $a_{1}$ is optimal if and only if $D_{n}(s, t) \geq 0$. Since

$$
F_{n}(s, t)=\max \left\{F_{n}^{1}(s, t), F_{n}^{2}(s, t)\right\},
$$


it is derived that

$$
F_{n}(s, t)=F_{n}^{2}(s, t)+\max \left\{F_{n}^{1}(s, t)-F_{n}^{2}(s, t), 0\right\}
$$

and

$$
\begin{gathered}
F_{n}(s, t)=F_{n}^{1}(s, t)+\max \left\{0,-F_{n}^{1}(s, t)+F_{n}^{2}(s, t)\right\} \\
=F_{n}^{1}(s, t)-\min \left\{F_{n}^{1}(s, t)-F_{n}^{2}(s, t), 0\right\}
\end{gathered}
$$

Therefore,

$$
F_{n}(s, t)=F_{n}^{2}(s, t)+D_{n}^{+}(s, t)
$$

and

$$
F_{n}(s, t)=F_{n}^{1}(s, t)-D_{n}^{-}(s, t)
$$

where

$$
D_{n}^{+}(s, t)=\max \left\{D_{n}(s, t), 0\right\}
$$

and

$$
D_{n}^{-}(s, t)=\min \left\{D_{n}(s, t), 0\right\} .
$$

Lemma 3 Let $s \geq 1$ and $t \geq 1$. Then

$$
\begin{gathered}
D_{2}(s, t)=2 \prod_{j=1}^{2} \frac{t+j-1}{s+t+j-1}-\frac{t}{s+t}, \\
D_{3}(s, t)= \begin{cases}2 \prod_{j=1}^{3} \frac{t+j-1}{s+t+j-1}-\frac{t}{s+t}, & \text { if } s \leq t+2, \\
-\frac{t}{s+t} \frac{s}{s+t+1}, & \text { if } t+2<s,\end{cases}
\end{gathered}
$$

and

$$
\begin{gathered}
D_{n}(s, t)=-\frac{t s}{(s+t)(s+t+1)}+\frac{t}{s+t} D_{n-1}^{+}(s, t+1)+\frac{t}{s+t} D_{n-2}^{-}(s, t+1) \\
+\frac{t s}{(s+t)(s+t+1)} D_{n-2}^{-}(s+1, t+1)
\end{gathered}
$$

for $n \geq 4$.

Proof. (4.9) is derived from (4.1) and (4.2), and (4.10) is derived from (4.5). For $n \geq 4$,

$$
\begin{aligned}
D_{n}(s, t)= & \frac{t}{s+t}\left(1+F_{n-1}(s, t+1)\right)-\frac{t}{s+t}\left(1+F_{n-2}(s, t+1)\right) \\
& -\frac{t s}{(s+t)(s+t+1)}\left(1+F_{n-2}(s+1, t+1)\right) .
\end{aligned}
$$

Substituting

$$
\begin{aligned}
& F_{n-1}(s, t+1)=F_{n-1}^{2}(s, t+1)+D_{n-1}^{+}(s, t+1), \\
& F_{n-2}(s, t+1)=F_{n-2}^{1}(s, t+1)-D_{n-2}^{-}(s, t+1),
\end{aligned}
$$

and

$$
F_{n-2}(s+1, t+1)=F_{n-2}^{1}(s+1, t+1)-D_{n-2}^{-}(s+1, t+1)
$$

into (4.12),

$$
D_{n}(s, t)=-\frac{t s}{(s+t)(s+t+1)}+\frac{t}{s+t} D_{n-1}^{+}(s, t+1)+\frac{t}{s+t} D_{n-2}^{-}(s, t+1)
$$




$$
\begin{aligned}
& +\frac{t s}{(s+t)(s+t+1)} D_{n-2}^{-}(s+1, t+1)+\frac{t}{s+t} F_{n-1}^{2}(s, t+1) \\
& -\frac{t}{s+t} F_{n-2}^{1}(s, t+1)-\frac{t s}{(s+t)(s+t+1)} F_{n-2}^{1}(s+1, t+1) .
\end{aligned}
$$

Also, substituting

$$
\begin{gathered}
F_{n-1}^{2}(s, t+1)=\frac{t+1}{s+t+1}\left(1+F_{n-3}(s, t+2)\right)+\frac{s(t+1)}{(s+t+1)(s+t+2)}\left(1+F_{n-3}(s+1, t+2)\right), \\
F_{n-2}^{1}(s, t+1)=\frac{t+1}{s+t+1}\left(1+F_{n-3}(s, t+2)\right)
\end{gathered}
$$

and

$$
F_{n-2}^{1}(s+1, t+1)=\frac{t+1}{s+t+2}\left(1+F_{n-3}(s+1, t+2)\right)
$$

into (4.13), (4.11) is easily derived after some calculations. This completes the proof. $\square$

Theorem 2 For $s>0, t>0$ and $n \geq 3$, if $D_{n}(s, t)>0$, then $D_{n-1}(s, t+1)>0$.

Proof. If $D_{3}(s, t)>0$, then it is derived from (4.10) that

$$
2 \prod_{j=1}^{3} \frac{t+j-1}{s+t+j-1}-\frac{t}{s+t}>0
$$

that is,

$$
2 \prod_{j=2}^{3} \frac{t+j-1}{s+t+j-1}-1>0
$$

from which

$$
\begin{aligned}
D_{2}(s, t+1) & =2 \prod_{j=1}^{2} \frac{(t+1)+j-1}{s+(t+1)+j-1}-\frac{(t+1)}{s+(t+1)} \\
& =2 \prod_{j=2}^{3} \frac{t+j-1}{s+t+j-1}-\frac{t+1}{s+t+1}>0 .
\end{aligned}
$$

For $n \geq 4$, it is derived from (4.11) that $D_{n}(s, t)>0$ means $D_{n-1}^{+}(s, t+1)>0$, that is, $D_{n-1}(s, t+1)>0$. This completes the proof. $\square$

If we consider the case of no accident as a win, then this theorem gives a stay-on-a-winner rule which plays an important role in the analysis of bandit problems (see for example, Berry and Fristedt [1]). In our problem, this rule is described as follows: If $a_{1}$ is optimal in state $(n ; s, t)$ and if $a_{1}$ is selected and no accident occurs, then $a_{1}$ is also optimal in the next state $(n-1 ; s, t+1)$.

Remark. In order to show that the adaptive backup strategy with learning is superior to the backup strategy without learning, we compare the value of $f_{3}(p)$ for the case of known value $p=2 / 7$ and the value of $F_{3}(s, t)$ for the case of unknown value of $p$ with $s=2$ and $t=5$, where $s /(s+t)=2 / 7$. Then,

$$
f_{3}\left(\frac{2}{7}\right)=\frac{545}{343}<\frac{5}{3}=F_{3}(2,5)
$$

This means that the backup strategy with learning is superior to the backup strategy without learning. 
Acknowledgements The author is grateful to the anonymous referees and Professor Tamaki, Aichi University, for their helpful comments. This reseach is supported in part by Grant-in-Aid for Scientific Research (B)(2) No.11680449 (1999-2000) of the Japanese Ministry of Education, Science, Sports and Culture, and also it is supported in part by the Special Grant-in-Aid for Research (1998) of Hyogo Prefecture.

\section{References}

[1] D. A. Berry and B. Fristedt: Bandit Problems: Sequential Allocation of Experiments. (Chapman and Hall, London and New York, 1985).

[2] K. M. Chandy and C. V. Ramamoorthy: Rollback and recovery strategies for computer programs. IEEE Transactions on Computer, C-21 (1972) 546-556.

[3] T. Dohi, T. Aoki, N. Kaio and S. Osaki: Computational aspects of optimal checkpoint strategy in fault-tolerant database management. IEICE Transactions on Fundamentals of Electronics, Communications and Computer Sciences E80-A (1997) 2006-2015.

[4] M. H. DeGroot: Optimal Statistical Decisions. (McGraw-Hill, New York 1970).

[5] S. Fukumoto, K. Yasui and T. Nakagawa: Data maintainability of the medium of a secondary storage with backup copies: An evaluation model and its application (in Japanese). The Transactins of the Institute of Electronics, Information and Communication Engineers A, J 77-A (1994), 680-686.

[6] E. Gelenbe: On the optimum checkpoint interval. Journal of Association on Computing Machinary, 2 (1979) 259-270.

[7] T. Hamada: Optimal backup strategy for making files by computer. Journal of the Operations Research Society of Japan, 41 (1998) 614-625.

[8] N. Kaio and S. Osaki: A note on optimum check pointing policies. Microelectron. Reliability, 25 (1985) 451-453.

[9] S. M. Ross: Introduction to Stochastic Dynamic Programming. (Academic Press, New York, 1983).

[10] H. Sandoh, N. Kaio and H. Kawaii: On backup policies for computer disks. Reliebility Engineering \& System Safety, 37 (1992) 1-9.

[11] H. Sandoh and H. Kawai: An optimal $N$-job backup policy maximizing availability for a hard computer disk. Journal of the Operations Research Society of Japan, 34 (1991) 383-390.

[12] H. Sandoh, H. Kawai and T. Ibaraki: An optimal backup policy for hard computer disk depending on age under availability criterion. Computers and Mathematics with Applications, 24 (1992) 57-62.

[13] S. Toueg and Ö. Babaog̃lu: On the optimum checkpoint selection problem. SIAM Journal of Computer, 13 (1984) 630-649.

[14] J. W. Young: A first order approximation to the optimum checkpoint interval. Communications of ACM, 17 (1974) 530-531.

Toshio Hamada

Department of Management Science

Kobe University of Commerce

Gakuen-nishimachi, Nishi-ku, Kobe 651-2197 JAPAN

E-mail: hamada@kobe.kobeuc.ac.jp 